\title{
A case report of bicornis bicollis uterus with unilateral cervical atresia: an unusual aetiology of chronic debilitating pelvic pain in a Cameroonian teenager
}

\author{
Julius Sama Dohbit ${ }^{1,2}$, Esther Meka ${ }^{1,2}$, Joel Noutakdie Tochie ${ }^{1 *}$, Igor Kamla ${ }^{1}$, Darolles Mwadjie ${ }^{3}$
} and Pascal Foumane $e^{1,2}$

\begin{abstract}
Background: Congenital uterine anomalies like bicornis or bicornuate uterus are relatively rare in sub-Saharan Africa. They are associated with an increased rate of spontaneous abortion, preterm delivery, and infertility. The occurrence of bicornis bicollis uterus with unilateral cervical atresia is exceptional and its management is controversial. We hereby report a rare cause of chronic pelvic pain in a Cameroonian teenager due to unilateral obstructive hematometra and hematosalpinx in the non-communicating horn of a bicornis bicollis uterus.

Case presentation: A 13-year-old premenarchal non-virgin female presented with chronic and severe cyclical crampy pelvic pain. On clinical examination, she had a perforated hymen, a single vagina, and one uterine cervix. A two-dimensional pelvic ultrasonography revealed hematometra but missed out the underlying anomaly. Failure to drain the hematometra by serial cervical dilatations prompted an exploratory laparotomy which revealed: bicornis bicollis uterus with a right rudimentary uterine horn communicating with the vagina and a left noncommunicating uterine horn distended by hematometra due to a homolateral cervical atresia. She underwent utero-vaginal canalization and a left hemi-hysterotomy with drainage of the hematometra. The postoperative period was uneventful. Regular cyclic menses occurred thereafter beginning at the first postoperative month. She had complete resolution of symptoms without recurrence after six months.

Conclusion: Due to the risk of compromised fertility from bicornis uterus and the diagnostic challenges akin to resource-limited settings, we highlight the need for a high index of suspicion by healthcare providers when faced with chronic pelvic pain in premenarchal adolescents.
\end{abstract}

Keywords: Bicornuate uterus, Bicornis bicollis uterus, Cervical atresia, Chronic pelvic pain, Cameroon

\section{Background}

Bicornuate uterus $(\mathrm{BU})$ is a congenital uterine anomaly with a prevalence of $0.4 \%$ in the general population [1]. It is often asymptomatic before puberty and thereafter has a significant association with infertility and miscarriage [1]. A bicornuate uterus consists of two symmetric uterine horns unified by caudal fusion. Both endometrial cavities communicate with the vagina either through a

\footnotetext{
* Correspondence: joeltochie@gmail.com

${ }^{1}$ Faculty of Medicine and Biomedical Sciences, University of Yaoundé I,

Yaoundé, Cameroon

Full list of author information is available at the end of the article
}

single uterine cervix (unicollis, most frequent) or through two uterine cervices (bicollis, less frequent). The occurrence of cervical atresia with $\mathrm{BU}$ is rare $[2,3]$. Patients may present with pelvic pain due to hematometra and retrograde menstruation from a non-communicating uterine horn [2]. Treatment options are controversial and include hysterectomy or utero-vaginal canalization with the principal goal of relieving the symptoms and preserving fertility $[3,4]$.

Contrary to high-income countries where epidemiological studies have been carried out on these anomalies [1], the relative rarity of BU in sub-Saharan Africa (SSA) 
is due to a low index of clinical suspicion; under-reporting and inaccessibility to highly sensitive diagnostic tests like magnetic resonance imaging (MRI) $[5,6]$. We herein, report an unusual case of bicornis bicollis uterus with unilateral cervical atresia in a Cameroonian teenager, presenting as chronic debilitating cyclical pelvic pain due to obstructive hematometra and managed by uterovaginal canalization.

\section{Case presentation}

A 13-year-old female student $\mathrm{G}_{0} \mathrm{P}_{0}$ presented with severe cyclical pelvic pain of gradual onset for a duration of 15 months. The pain was constricting in nature, predominant in the left iliac fossa, radiating to the back and perianal region and unremitting to analgesics and antispasmolytics. There was no fever, no urinary or gastrointestinal symptom. She had interrupted her education eight months prior to consultation due to the incapacitating nature of the pain.

She was premenarchal, had pubarche and thelarche at the ages of 9 and 11 years respectively. She had no chronic illness and had never undergone surgery. Her psychosocial and family histories were unremarkable. She had first coitus at the age of 11 years.

On examination, she was ill looking and had Tanner stage IV secondary sexual characteristics. Her temperature was $38.1{ }^{\circ} \mathrm{C}\left(100.58{ }^{\circ} \mathrm{F}\right)$ and other vital signs were within normal limits. Her conjunctivae were not pale. Abdominal examination revealed tenderness at the supra-pubic region and left iliac fossa without guarding. A moderately tender, rubbery, roughly spherical, supra-pubic pelvic mass of about $7 \mathrm{~cm}$ (longest diameter) was palpable. The physical appearance of her external genitalia was normal and she had a perforated hymen. On speculum examination, the vagina and cervix were normal. There was mild cervical motion tenderness, the pouch of Douglas was empty and the uterus was of normal size. Supra-pubic tenderness hindered clinical evaluation of the adnexae. Digital rectal examination and other systemic examinations were non-contributory. A working diagnosis of cervical stenosis complicated by cryptomenorrhoea was made and as differential diagnoses: chronic pelvic inflammatory disease and a pathologic ovarian cyst were advocated.

A two-dimensional pelvic ultrasound scan demonstrated the presence of hydrometra of $225 \mathrm{ml}$ (Fig. 1) and bilateral hydrosalpinx more predominant on the left side. She was admitted for an ultrasound guided cervical dilation under general anaesthesia. This procedure failed to drain any intra-uterine fluid despite easy intrauterine access with Hegar dilators. An intra-intervention ultrasound scan revealed the presence of a uterus of normal size and two lateral uterine masses. In the absence of an MRI scan in our setting to further explore the pelvis and these masses, the patient and her guardians were counselled

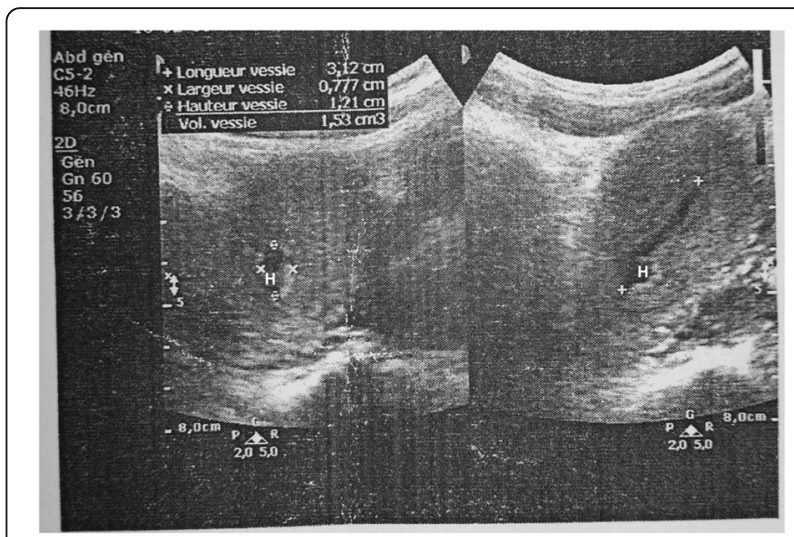

Fig. 1 Showing Hydrometra $(H)$ on two-dimensional pelvic ultrasound scan

and they consented to an exploratory laparotomy. Intra-operative findings were; a bicornis bicollis uterus with a left non-communicating uterine horn distended by hematometra due to left sided cervical atresia; a right atrophic rudimentary uterine horn communicating with the unique vagina (Fig. 2). Each horn had one ovary, one fallopian tube, one round ligament and one utero-sacral ligament. Both horns were separated at their base by a septum. The left ovary was hypertrophic and congested, while the right ovary was grossly normal. The left fallopian tube was tortuous and distended by blood, suggestive of hematosalpinx. Many tubo-uterine and tubo-ovarian adhesions were present. No other abdominal malformation was observed. Intra-operative management consisted of a left vertical corporeal hemi-hysterotomy of about $2 \mathrm{~cm}$, which ensured drainage of approximately $300 \mathrm{ml}$ of a tarry brown intra-uterine fluid (Fig. 3). The left uterine cavity was probed with Hegar's dilators until felt through a fibrosed transverse membrane over the blind pouch into the vagina. The membrane was incised posteriorly, creating an ostium between the vagina and the left uterine cavity. A foley

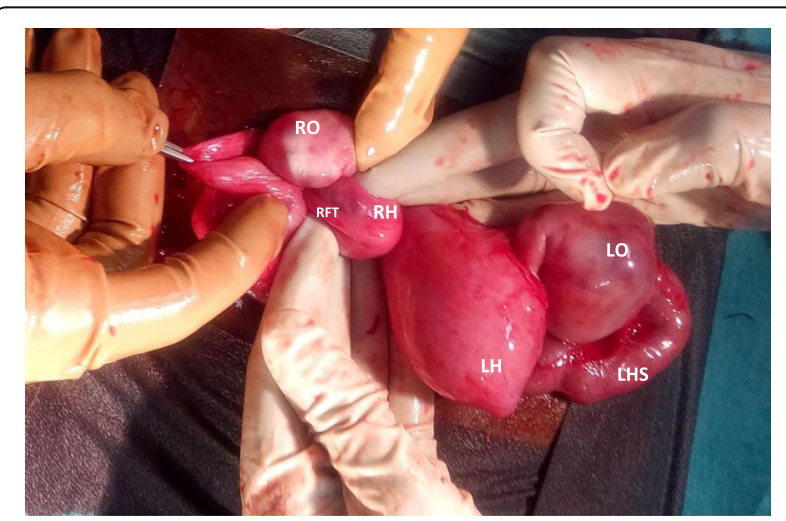

Fig. 2 Bicornuate uterus with a left distented uterine horn $(\mathrm{LH})$, right rudimentary uterine horn $(\mathrm{RH})$, left hematosalpinx (LHS), right fallopian tube (RFT), congested left ovary (LO) and right ovary (RO) 


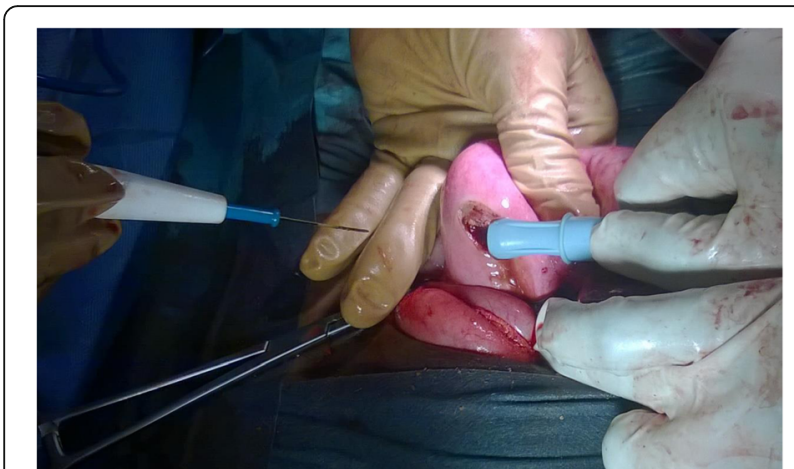

Fig. 3 Left vertical corporeal hemi-hysterotomy and suction of the hematometra

catheter was placed in the uterovaginal canal as a "stent". The hysterotomy incision was closed in two layers. Both ovaries were conserved. The peritoneal cavity was washed with warm saline and the anterior abdominal wall closed layer by layer. The postoperative period was uneventful. The uterine catheter was removed on the fifth postoperative day and the patient was discharged on the sixth day with plans of follow-up cervical dilatation procedures. A postoperative abdominal ultrasound scan ruled out renal anomalies. She started regular monthly menstruation from the first postoperative month without dysmenorrhoea. She had complete resolution of symptoms without recurrence after six months.

\section{Discussion}

Congenital uterine anomalies are incidental findings. A recent systematic review showed a prevalence of $5.5 \%$ in the general population, with a prevalence rate of $0.2 \%$ $0.6 \%$ accounted by BU [1]. BU arises from an incomplete lateral fusion of the two Mullerian ducts at about the tenth week of intrauterine life from idiopathic causes [1]. The association of BU with cervical atresia is unusual because $\mathrm{BU}$ is often a non-obstructive type of Mullerian anomaly. Only sporadic cases similar to this one have been reported along with their surgical management [2,3]. The occurrence of primary amenorrhoea associated with normal secondary sexual characteristics and cyclical pelvic pain (that worsens during puberty) should result in consideration of an obstructive congenital anomaly of this sort $[7,8]$. Pelvic examination helps to rule out an imperforated hymen or a blind vaginal pouch, and may reveal a pelvic mass corresponding to hematometra, or hematosalpinx resulting from obstructed menstrual flow [8, 9]. Alternatively, patients may be asymptomatic in case the uterine horns are lined with nonfunctional endometrium [10] or symptoms may be nonspecific, leading to late diagnoses and a high incidence of complications [11]. The resultant cyclical pelvic pain may be non-responsive to analgesics and severe enough to interfere with the patient's quality of life, as seen in the above case [12, 13]. Our low index of clinical suspicion owing to the scarcity of data on the epidemiology and clinical presentation of this anomaly in SSA, coupled with sub-optimal diagnostic testing (two-dimensional ultrasonography) illustrate the frequent diagnostic challenges of congenital uterine anomalies encountered in resource-limited settings [5]. Three-dimensional ultrasound is increasingly being preferred over two-dimensional ultrasound for diagnosis because it provides a coronal view that enables an accurate description of the cervical anatomy as well as the differentiation and morphological classification of the various subtypes of Mullerian duct anomalies [14]. However, the high power of resolution of MRI makes it the imaging technique of choice for the diagnosis of uterine anomalies, its associated urinary tract malformations and in choosing the most appropriate surgical technique $[8,15]$. Our patient could not benefit from an MRI scan because this sophisticated and expensive diagnostic test was inaccessible to her at the time of her management. At present, Cameroon has only three functional MRI machines for about 22 million inhabitants.

The treatment of BU with cervical atresia is controversial, consisting of surgical techniques like hysterectomy or utero-vaginal canalization $[3,4]$. The latter is conservative surgery that entails the creation of a new endocervical canal through the fibrous tissues separating the uterus from the vagina, followed by putting in place of a stent often with a surrounding full or split-thickness skin graft to sustain epithelial ingrowth [4]. Historically, due to the high postoperative mortality rate and complications of utero-vaginal canalization, namely re-stenosis of the neo-ostium, low postoperative fertility rate and severe infection by ascending route, some authors advocate for hysterectomy even in young patients $[2,16]$. However. utero-vaginal canalization is now being frequently performed due to recently reported uneventful postoperative outcomes, successful term deliveries after surgery and reduced psychological sequelae [4, 8, 17]. Some authors recommend that this surgery should be performed before puberty to avoid the risk of endometriosis from retrograde menstruation $[8,18]$. Together with the patient's family, we chose to be conservative and we performed uterovaginal canalization considering the strong future pregnancy desires. Generally, the main aims of treatment are to relieve symptoms, to provide a conduit for regular menstruation and to preserve reproductive potential [7]. In our case, the short-term goal of treatment was attained; resolution of pelvic pain, initiation of regular menstruation (ruling out re-stenosis of the new cervical canal) and the absence of post-operative infection when she was last re-evaluated at the sixth postoperative month. Regular follow-up visits are however, recommended for early detection of any late postoperative complication. 
Finally, fertility prognosis is debatable as successful term pregnancies by natural or artificial insemination have been reported after surgical correction $[8,10,19]$. However, fertility may be compromised by several other factors including the lack of cervical mucus, upper reproductive tract malformations, restenosis of the new cervix, postoperative adhesions and endometriosis caused by retrograde menstruation prior to surgery $[7,10,20,21]$. Grimbizis et al. observed an overall $55.2 \%$ live birth rate, $40.6 \%$ term delivery rate, $36 \%$ spontaneous abortion rate, and $23 \%$ preterm birth rate in 261 women with BU [22]. Although there is currently no randomised controlled trial in favour or against surgical resection of the non-communicating horn of a Mullerian duct anomaly prior to conception in women with poor reproductive performances, several publications in the form of case reports and case series have shown successful postoperative term pregnancies following surgical resection of this uterine horn, preferably performed via laparoscopy [23-25]. Furthermore, resection of the non-communicating horn is often indicated if it is lined with functional endometrium, in order to avert catastrophic complications of horn pregnancies, ectopic pregnancies and endometriosis [26]. Hence in our case, resection of the right rudimentary horn was not absolutely indicated because of it was thought to be line with non-functional endometrium, given its absence of menstrual bleeding despite communication with the vagina. On the other hand, surgical resection of the left non-communicating uterine horn which presented with obstructive symptoms was indicated. However, because this horn carried the sole apparent probability of hosting a pregnancy in the present nulliparous patient, its resection was not performed due to refusal by her family. The patient and her family were counselled on the importance of regular clinical follow-up visits and to seek appropriate qualified obstetric evaluation in due course when pregnancy is desired.

\section{Conclusion}

We have reported the first case of concomitant bicornis bicollis uterus with unilateral cervical atresia in Cameroon. Although limited resources precluded proper investigation with magnetic resonance imaging, we have shown that utero-vaginal canalization could yield favourable postoperative outcomes. This would need to be further explored in large multi-centre clinical trials in our setting. Health care personnel should have a high index of clinical suspicion for this congenital uterine anomaly as a potential differential diagnosis in premenarchal Cameroonian adolescents with unremitting cyclical chronic pelvic pain. Robust and advanced imaging modalities when available should help confirm clinical suspicion. The benefits of a timely diagnosis and treatment of such congenital uterine anomalies cannot be overemphasized in a setting already burdened with elevated female infertility.
Abbreviations

BU: Bicornuate Uterus; MRI: Magnetic resonance imaging; SSA: Sub-Saharan Africa

\section{Acknowledgments}

We are grateful to Drs Vitalis Fambombi Feteh and Tsi Njim for proofreading this article.

Availability of data and materials

All data generated during this study are included in this case report.

Funding

None.

Authors' contributions

JSD, EM, JNT, IK, DW: management of the patient, acquisition of data, manuscript writing and revisions. PF: acquisition of data, manuscript writing and critical revisions. All the authors read and approved the final manuscript.

Competing interests

The authors declare that they have no competing interests.

\section{Consent for publication}

Written informed consent was obtained from the parents of the patient for publication of this case report and accompanying images.

Ethics approval and consent to participate

Not applicable.

\section{Author details}

${ }^{1}$ Faculty of Medicine and Biomedical Sciences, University of Yaoundé I, Yaoundé, Cameroon. 'Department of Obstetrics and Gynaecology, Gynaeco-Obstetric and Paediatric Hospital, Yaoundé, Cameroon. ${ }^{3}$ Department of Obstetrics and Gynaecology, Sangmelima Reference Hospital, Sangmelima, Cameroon.

Received: 5 October 2016 Accepted: 24 May 2017

Published online: 02 June 2017

\section{References}

1. Chan YY, Jayaprakasan K, Zamora J, Thornton JG, Raine-Fenning N, Coomarasamy A. The prevalence of congenital uterine anomalies in unselected and high-risk populations: a systematic review. Hum Reprod Update. 2011;17(6):761-71.

2. Gasim T, Al Jama FE. Massive Hematometra due to congenital Cervicovaginal agenesis in an adolescent girl treated by hysterectomy: a case report. Case rep Obstet Gynecol. 2013;2013:1-3.

3. Xie Z, Zhang X, Liu J, Zhang N, Xiao H, Liu Y, et al. Clinical characteristics of congenital cervical atresia based on anatomy and ultrasound: a retrospective study of 32 cases. Eur J med res. 2014;19(1):10.

4. Gurbuz A, Karateke A, Haliloglu B. Abdominal surgical approach to a case of complete cervical and partial vaginal agenesis. Fertil Steril. 2005;84(1):217. e9-217.e11.

5. Feteh VF, Dimala CA, Njim T, Fuka B. Post term pregnancy in a non-communicating rudimentary horn of a unicornuate uterus. BMC res Notes. 2016;9:209.

6. Nwosu BO, Ugboaja JO, Obi-Nwosu A. Spontaneous rupture of gravid horn of Bicornuate uterus at term - a case report. Niger med J. 2010;51(4):184-5.

7. Radhouane A, Mohamed B, Imen BA, Aymen F, Khaled N. Successful pregnancy by IVF in a patient with congenital cervical atresia. Asian Pac J Reprod. 2015:4(3):249-50.

8. Acien P, Acien MI, Quereda F, Santoyo T. Cervicovaginal agenesis: spontaneous gestation at term after previous reimplantation of the uterine corpus in a neovagina: case report. Hum Reprod. 2008;23(3): 548-53.

9. Fujimoto VJ, Miller JH, Klein NA, Soules MR. Congenital cervical atresia: report of seven cases and review of the literature. Am J Obstet Gynecol. 1997:177:1419-25

10. Deffarges JV, Haddad B, Musset R, Paniel BJ. Utero-vaginal anastomosis in women with uterine cervix atresia: long-term follow-up and reproductive performance. A study of 18 cases. Hum Reprod. 2001;16:1722-5.

11. Liatsikos SA, Tsikouras P, Souftas V, Ammari A, Prassopoulos P, Tsikos SA, et al. Diagnosis and laparoscopic management of a rudimentary uterine horn in a 
teenage girl, presenting with haematometra and severe endometriosis: our experience and review of literature. Minim Invasive Ther Allied Technol. 2010; 19(4):241-7.

12. Dwivedi $R$, Perera $K$, Eedarapalli $P$. Non-communicating inactive rudimentary horn of the uterus presenting with dysmenorrhoea- a case report of successful laparoscopic excision. Open J Obstet Gynecol. 2011;01(04):213-6.

13. Badawy SZA, Montgomery S. Severe primary Dysmenorrhea due to functioning Noncommunicating rudimentary horn. J Gynecol Surg. 2009;25(1):23-7.

14. Woelfer B, Salim R, Banerjee S, Elson J, Regan L, Jurkovic D. Reproductive outcomes in women with congenital uterine anomalies detected by threedimensional ultrasound screening. Obstet Gynecol. 2001;98(6):1099-103.

15. Marten K, Vosshenrich R, Funke M, Obenauer S, Baum F, Grabbe E. MRI in the evaluation of müllerian duct anomalies. Clin Imaging. 2003:27(5):346-50.

16. Casey AC, Laufer MR. Cervical agenesis: septic death after surgery. Obstet Gynecol. 1997:90(4 Pt 2):706-7.

17. Shah TN, Venkatesh S, Saxena RK, Pawar S. Uterovaginal anastomosis for complete cervical agenesis and partial vaginal agenesis: a case report. Eur J Obstet Gynecol Reprod Biol. 2014;174:154-5.

18. Bugmann P, Amaudruz M, Hanquinet S, La Scala G, Birraux J, Le Coultre C. Uterocervicoplasty with a bladder mucosa layer for the treatment of complete cervical agenesis. Fertil Steril. 2002;77:831-5.

19. Chakravarty B, Konar $\mathrm{H}$, Chowdhury NN. Pregnancies after reconstructive surgery for congenital cervicovaginal atresia. Am J Obstet Gynecol. 2000; 183:421-3.

20. Aimen FM, Atef Y, Majed G, Radhouane A, Manel M, Monia M, et al. Spontaneous pregnancy after vaginoplasty in a patient presenting a congenital vaginal aplasia. Asian Pac J Reprod. 2016;5(4):351-3.

21. Matalliotakis LM, Goumenou AG, Matalliotakis M, Arici A. Uterine anomalies in women with endometriosis. J Endometr. 2010;2(4):213-7.

22. Grimbizis GF, Camus M, Tarlatzis BC, Bontis JN, Devroey P. Clinical implications of uterine malformations and hysteroscopic treatment results. Hum Reprod Update. 2001;7(2):161-74.

23. Caserta D, Mallozzi M, Meldolesi C, Bianchi $P$, Moscarini M. Pregnancy in a unicornuate uterus: a case report. J med Case rep. 2014;8:130

24. Kim T-H. Laparoscopic resection of the rudimentary horn of a unicornuate uterus diagnosed by three-dimensional computed tomography. Med Case Stud. 2013:4(1):9-12

25. Spitzer RF, Kives S, Allen LM. Case series of laparoscopically resected noncommunicating functional uterine horns. J Pediatr Adolesc Gynecol. 2009;22(1):e23-8.

26. Atmaca R, Germen AT, Burak F, Kafkasli A. Acute abdomen in a case with noncommunicating rudimentary horn and unicornuate uterus. JSLS. 2005; 9(2):235-7.

\section{Submit your next manuscript to BioMed Central and we will help you at every step:}

- We accept pre-submission inquiries

- Our selector tool helps you to find the most relevant journal

- We provide round the clock customer support

- Convenient online submission

- Thorough peer review

- Inclusion in PubMed and all major indexing services

- Maximum visibility for your research

Submit your manuscript at www.biomedcentral.com/submit 